\title{
Multi-kinase Inhibitor TPX-0022
}

National Cancer Institute

\section{Source}

National Cancer Institute. Mult-kinase Inhibitor TPX-0022. NCI Thesaurus. Code

C162676.

An orally bioavailable, multi-targeted kinase inhibitor with potential antineoplastic activity. Upon oral administration, multi-kinase inhibitor TPX-0022 binds to and inhibits three tyrosine kinases that are often overexpressed in a variety of cancer cell types, including MET (c-Met; hepatocyte growth factor receptor; HGFR) , Src, and colony stimulating factor 1 receptor (CSF1R; CSF-1R; C-FMS; CD115; macrophage colony-stimulating factor receptor; M-CSFR) thereby disrupting their respective signaling pathways. MET, a receptor tyrosine kinase overexpressed or mutated in many tumor cell types, plays an important role in tumor cell proliferation, survival, invasion, and metastasis, and in tumor angiog enesis. Src, a non-receptor tyrosine kinase upregulated in many tumor cell types, plays an important role in tumor cell proliferation, motility, invasiveness and survival. CSF1R is a cell-surface receptor for colony stimulating factor 1 (CSF1); this receptor tyrosine kinase is overexpressed by tumor-associated macrophages (TAMs) in the tumor microenvironment (TME), and plays a major role in both immune suppression and the induction of tumor cell proliferation. 